\title{
Silk inverse opals from template-directed $\beta$-sheet transformation of regenerated silk fibroin $\dagger$
}

\author{
Victoria M. Swinerd, ${ }^{a}$ Andrew M. Collins, ${ }^{a}$ Nicholas J. V. Skaer, ${ }^{b}$ Tom Gheysens ${ }^{b}$ and Stephen Mann*a
}

Received 7th August 2007, Accepted 17th September 2007

First published as an Advance Article on the web 27th September 2007

DOI: 10.1039/b711975e

We describe a novel and facile method for the fabrication of reconstituted silk monoliths with ordered interconnected air spheres based on intercalation and in situ $\beta$-sheet transformation of regenerated silk fibroin solutions within the interstitial spaces of a sacrificial colloidal crystal template. The silk inverse opals are elastic and can withstand and recover from compressive loads of up to $112 \mathrm{MPa}$ by reversible pore deformation. They also exhibit super-hydrophobicity with water droplet contact angles of up to $140^{\circ}$ due to periodic nanoscale protrusions associated with the surface texture of the inverse opal architecture. These properties indicate that silk inverse opals could have potential applications as biocompatible elastic scaffolds, storage-release, barrier and self-cleaning materials, and in the design of load-responsive microfluidic devices.

Biologically derived materials are a resource and inspiration for the development of advanced functional polymers, composites and nanostructures. ${ }^{1}$ In particular, there has been widespread interest in the use of silk, a protein-based polymer with remarkable mechanical properties, ${ }^{2}$ for a variety of novel materials-based applications. For example, native silk fibres have been used to control the deposition of inorganic materials such as magnetite, gold and cadmium sulfide, ${ }^{3}$ silica, ${ }^{4}$ or titania and zirconia $^{5}$ to produce composite materials with multiple functionality. In addition, reconstituted silks in the form of fibres, ${ }^{6}$ microspheres ${ }^{7,8}$ and scaffolds for tissue engineering ${ }^{9-11}$ have been prepared using regenerated or genetically engineered silk proteins (fibroins), but such procedures have not been exploited for the development of 3D patterned silk materials. Regenerated (redissolved) fibroins predominantly consist of partially degraded silk proteins in $\alpha$-helical and random coil conformations, and as such appear to be promising starting materials for transformation into elastomeric silk matrices by promotion of the $\beta$-sheet conformation. Formation of the $\beta$-sheet secondary structure, for example by treatment of fibroin solutions with solvents such as ethanol, results in enhanced inter-chain hydrogen bonding and nucleation of well-ordered crystalline domains separated by less crystalline $\alpha$-helical regions that deform elastically. This approach is to some extent compromised by the mechanical properties of the reconstituted silks, which often do not match those of the native

${ }^{a}$ Centre for Organized Matter Chemistry, School of Chemistry,

University of Bristol, Bristol, UKBS81TS.E-mail: s.mann@bris.ac.uk ${ }^{b}$ Oxford Biomaterials Ltd, Units 14-15 Galaxy House, New Greenham Business Park, Thatcham, UK RG19 6HR

$\uparrow$ Electronic supplementary information (ESI) available: SEM images of silk inverse opals prepared using regenerated fibroin solutions. See DOI: $10.1039 / \mathrm{b} 711975 \mathrm{e}$ biomaterial due to chemical degradation of the constituent proteins during processing. ${ }^{12,13}$

In general, it has proved difficult to produce patterned materials from regenerated silk proteins due to the inherent metastability of fibroin solutions during processing. Here, we present a method to produce three-dimensionally ordered macroporous reconstituted silk monoliths using the process of colloidal crystal templating. ${ }^{14}$ Silk inverse opals were prepared by capillary infiltration of preformed colloidal crystals of $500 \mathrm{~nm}$-diameter polystyrene microspheres with an aqueous $8 \%$ wt solution of regenerated silkworm (Bombyx mori) silk fibroin.t Opals prepared from polystyrene beads were used specifically to promote favourable interactions with the largely hydrophobic sequence domains of fibroin, ${ }^{15}$ and slow capillary ingress undertaken to prevent premature shear force-induced $\beta$-sheet formation during fabrication. The infiltrated samples were air dried, and then treated with ethanol for one hour to induce $\beta$-sheet formation and concomitant deposition of a reconstituted silk matrix within the interstitial spaces of the colloidal crystal template. Removal of the polystyrene template by washing in toluene produced a monolithic replica in the form of a silk inverse opal (Fig. 1(a)). High magnification SEM images confirmed high fidelity replication of periodic arrays of spherical voids that were enclosed within thin walls of silk (Fig. 1(b)). The replicas consisted of domains of close-packed $400 \mathrm{~nm}$-diameter spherical pores that were interconnected through $150 \mathrm{~nm}$-wide apertures and surrounded by a continuous silk-matrix framework, ca. $50 \mathrm{~nm}$ in thickness. The pore diameter was slightly less than the mean size of the polystyrene beads, possibly due to contraction of the matrix when exposed to the vacuum of the SEM. Similar results were obtained using regenerated fibroin solutions at concentrations of 4 and $6 \%$ wt except that the wall thicknesses were reduced to approximately $40 \mathrm{~nm}$ (see ESI $\dagger$, Fig. S1(a and b)). Replicas prepared using $2 \%$ wt fibroin solutions showed extensive fragmentation due to incomplete wall formation particularly at the contact edges between adjacent void spaces (see ESI $\dagger$, Fig. S1(c)).

Confirmation of $\beta$-sheet formation in the macroporous silk scaffold was determined by FTIR spectroscopy, which showed amide $\mathbf{I}(\mathrm{C}=\mathrm{O}$ str) and II (N-H bend) absorbance peaks at 1628 and $1526 \mathrm{~cm}^{-1}$, respectively (Fig. 2). The presence of amide III (C-N str) peaks at $1233 \mathrm{~cm}^{-1}$ and $1260 \mathrm{~cm}^{-1}$ indicated that the reconstituted silk matrix contained a mixture of disordered $\alpha$-helical and crystalline $\beta$-sheet domains. ${ }^{16}$ This was also consistent with the presence of a shoulder peak at $1700 \mathrm{~cm}^{-1}$ close to the amide $\mathbf{I}$ absorbance, which was associated with a $\beta$-turn or intermediate $\beta$-sheet structure. ${ }^{17,18}$

Elastic deformation properties of the silk inverse opal were determined by SEM imaging of samples maintained at different 


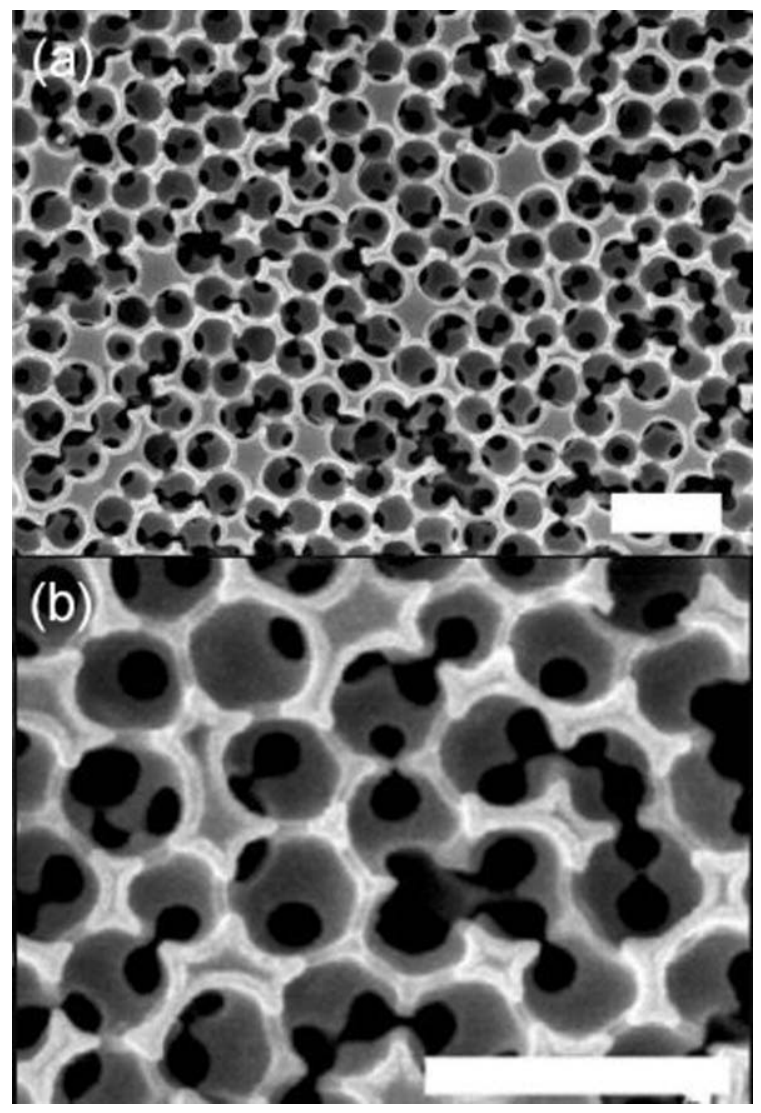

Fig. 1 SEM images of a silk inverse opal prepared using $8 \%$ wt regenerated fibroin, (a) low magnification, and (b) high magnification. Scale bars $=1 \mu \mathrm{m}$.

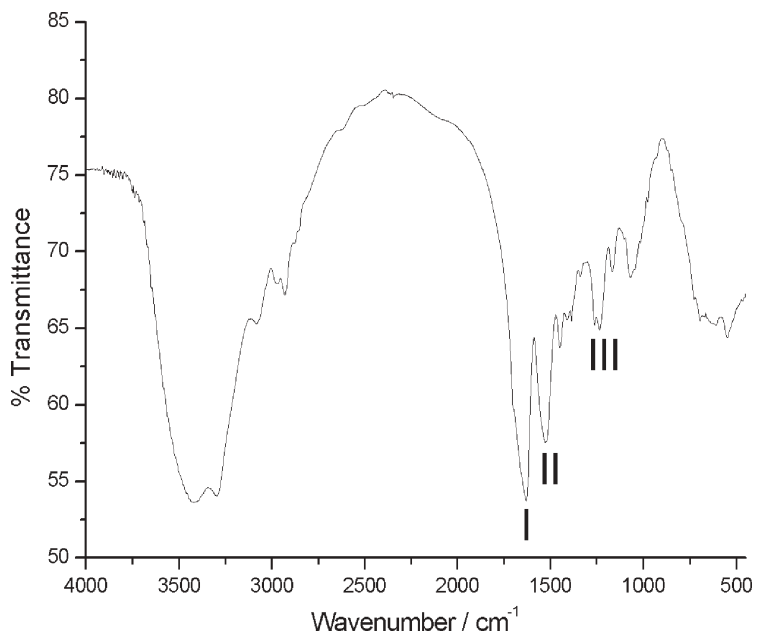

Fig. 2 FTIR spectrum of silk inverse opal showing amide I, II and III absorbances (see text for details).

levels of compression.§ Under compression loads of less than $112 \mathrm{MPa}$, the samples showed retention of the inverse opal structure with few failure points and consistent stress distribution (Fig. 3(a)). At relatively small deformations, the linear relationship between the applied force, $F$, and resultant deformation, $d$, is given by; $F=\left(k E t^{2} d\right) / /\left[12\left(1-\sigma^{2}\right)\right]$, where $t$ is the monolith thickness, $E$ the Young's modulus, $\sigma$ the Poisson ratio (transverse contraction

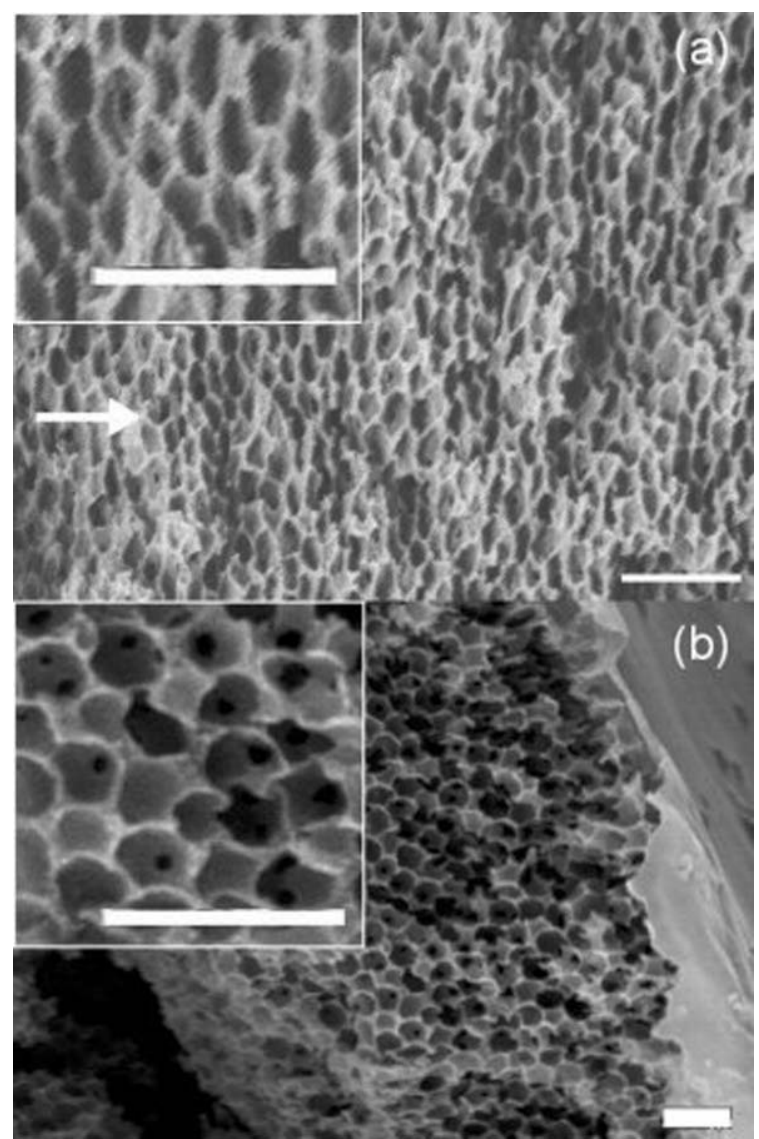

Fig. 3 SEM images of a silk inverse opal, (a) under compression (54 $\mathrm{MPa}$ ) showing anisotropic pore deformation (white arrow indicates direction of applied load), and (b) after compression at $54 \mathrm{MPa}$ showing isometric voids due to elastic relaxation. Insets show corresponding high magnification images of selected areas. Scale bars $=1 \mu \mathrm{m}$.

strain/longitudinal extension strain; $\varepsilon_{\mathrm{T}} / \varepsilon_{\mathrm{L}}$ ), and $k=$ constant. ${ }^{19}$ Under these conditions, the pore shape deformation increased from $R$ values of $c a$. 1.0 (unloaded) to $1.7(112 \mathrm{MPa})$, where $R=$ pore length/width, and was approximately proportional to the compressive strength (Fig. 4). Thus, the observed behaviour was consistent with a linear elastic inverse opal, in which changes in the pore length and width were analogous to the Poisson ratio deformations observed for solid materials. Significantly, when the compressive load was removed, the deformed pore structures relaxed back to $R$ values of around 1.1, i.e. close to the isometric condition observed for the non-loaded materials (Fig. 3(b) and 4), confirming that the silk inverse opal was elastomeric. Increasing the load above $112 \mathrm{MPa}$ up to a value of $c a .250 \mathrm{MPa}$ resulted in progressively increased fragmentation of the silk replicas although localized regions remained intact with pore deformations rising to $R$ values of around 1.85 . These regions subsequently relaxed into spherical voids when the load was removed. The plateau in $R$ values was thus consistent with the onset of material failure of the elastic monolith at high compression loads.

Wetting properties of the silk inverse opals were determined by measuring the contact angle of water droplets applied to the sample surface. Compared with a textureless film of reconstituted silk, which exhibited contact angles of less than $40^{\circ}$, the macroporous silk monoliths showed dramatically different behaviour 


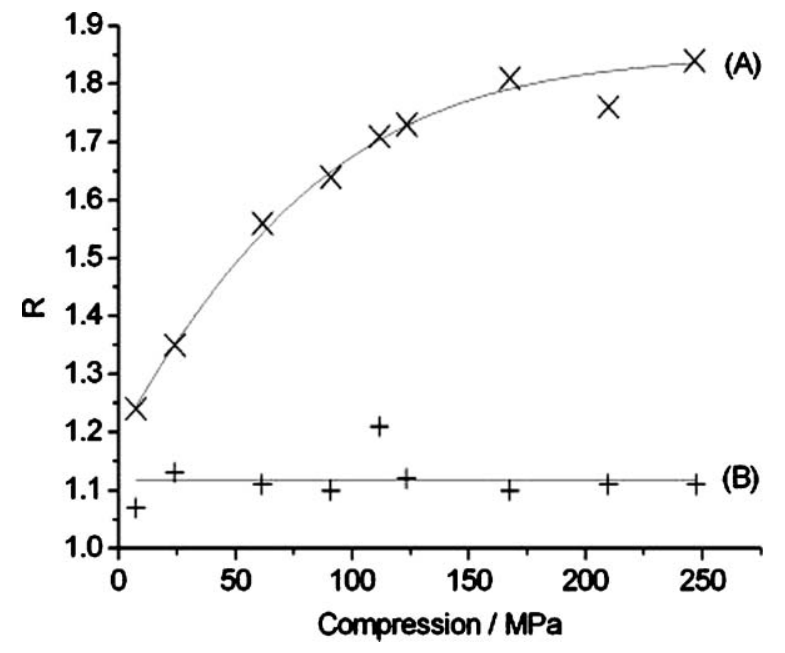

Fig. 4 Plots showing extent of pore deformation in a silk inverse opal ( $R=$ pore length/pore width) during (A) and after (B) compression at various applied loads.

with contact angles greater than $100^{\circ}$ and up to $140^{\circ}$ (Fig. 5). The results indicated a highly enhanced hydrophobicity associated with the nanoscale texture of the inverse opal surface, and were consistent with observations of "super-hydrophobicity" recorded for textured biological or synthetic surfaces comprising nanoscopic protrusions. ${ }^{20,21}$ In such cases, the surface nanostructure significantly increases the energy required for a liquid to overcome the interfacial surface tension upon wetting, such that when this energy requirement is larger than the surface tension the liquid remains as a droplet suspended upon the surface. ${ }^{22}$

In conclusion, we have used polystyrene colloidal crystals to spatially organize a regenerated silk-fibroin solution such that in situ transformation to the crystalline $\beta$-sheet state results in a macroporous silk inverse opal. The void dimensions and connectivity are replicated with high fidelity to produce an ordered reconstituted silk scaffold with good retention of the elastic properties, such that the monoliths are capable of spreading load

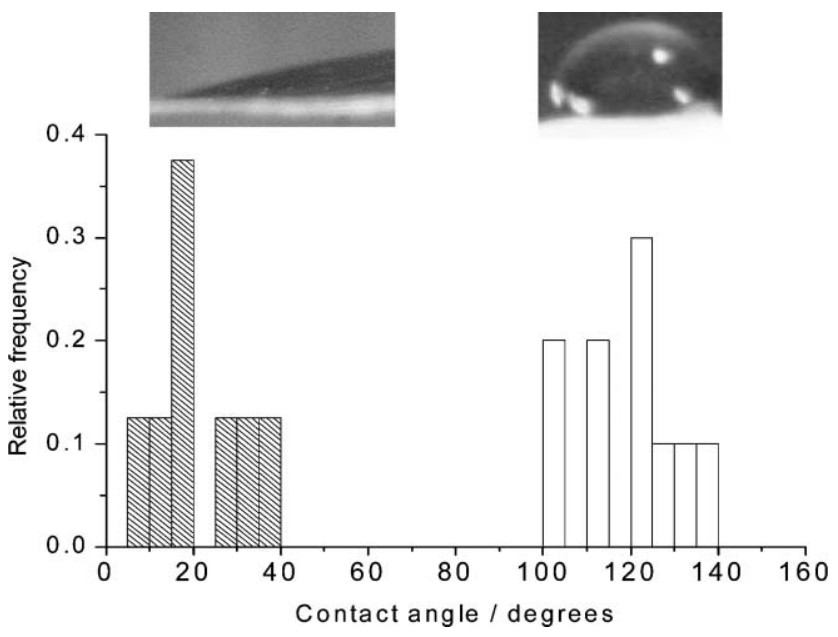

Fig. 5 Contact angle values for water droplets on non-porous silk film (hatched bars), and silk inverse opal (open bars). Insets show corresponding optical microscope images of water droplets. evenly by a reversible process involving anisotropic pore deformation. The replicas could therefore have uses in synthetic or medical applications that require tough biocompatible materials of loadbearing capability and elasticity. Moreover, the ability of these materials to undergo reversible compression by anisotropic pore deformation might be useful in microfluidic devices, for incorporating strength and biocompatibility in a hydrophobic proteinbased mixing chamber for example, or for controlling flow properties through a scaffold via reversible distortions in the shape of the internal pores. Finally, as the inverse opal architecture of the reconstituted silk monoliths significantly reduces surface wettability, such materials would be expected to show enhanced barrier and self-cleaning properties.

\section{Acknowledgements}

This work was supported by the EPSRC (V.M.S) and by Oxford Biomaterials (OBM) Ltd. (A.M.C).

\section{Notes and references}

† Regenerated Bombyx mori silk fibroin (degummed) was purchased from Oxford Biomaterials Ltd. and used as supplied. All other reagents were laboratory grade. Silica substrates were prepared by washing in $\mathrm{H}_{2} \mathrm{SO}_{4}$ $\mathrm{H}_{2} \mathrm{O}_{2}$ (80:20) overnight followed by consecutive 15 minute soakings in $20 \mathrm{~mL}$ of poly(allylamine hydrochloride) (PAH), poly(sodium 4-styrenesulfonate) (PSS) and PAH again, to yield a net positive charge on the silica surface that facilitated removal of the colloidal crystals. Colloidal crystals were grown on the modified silica substrates under vacuum from $50 \mathrm{~mL}$ of an ethanolic suspension of $500 \mathrm{~nm}$-diameter polystyrene microspheres (1\%wt). Portions of the colloidal crystals $\left(c a .1 \mathrm{~mm}^{2}\right)$ were detached from the substrate and carefully placed onto the surface of a silk-fibroin solution $(2-8 \% \mathrm{wt})$, and left in sealed vials for 4 days at room temperature to allow complete infiltration of the fibroin solution by capillary action. Infiltration using regenerated fibroin at concentrations greater than $8 \%$ wt was less successful due to the increased viscosity of the protein solutions. The impregnated samples were removed using tweezers, air dried, and then treated with ethanol for one hour to induce $\beta$-sheet formation and concomitant deposition of the silk matrix within the interstitial spaces of the colloidal crystal template. The polystyrene template was then removed by washing in toluene for one hour to yield a silk inverse opal.

$\S$ A compression device comprising two stainless steel plates $(25 \times 10 \times$ $5 \mathrm{~mm}$ ) held together in parallel using two screws $(20 \mathrm{~mm})$ was fabricated. Quantitative force measurements were obtained by placing samples of the silk inverse opal between the stainless steel plates and compressing using an Imada digital force gauge DPS-50R. Outputs were recorded in kilograms and converted to applied pressure, and then normalised to the surface area of the silk samples. Loads between 7 and $247 \mathrm{MPa}$ were used. Compression was maintained in isolation from the force gauge by tightening of the screws on the compression device. The device was then inserted into the $32 \mathrm{~mm}$-diameter sample holder of a SEM for direct imaging under compression.

- Water droplets $(0.4 \mu \mathrm{L}$, MilliQ) were used to wet the silk inverse opals samples and contact angles measured using a reconfigured optical microscope (Zeiss, Stemi SV11). The stage was removed and the microscope re-positioned such that the incident beam ran parallel to the horizontal. An adjustable platform was positioned in place of and at 90 degrees to the original stage, and an adjustable volume pipette was positioned directly above the focal point, which was illuminated by a fibreoptic light. A digital camera (Canon EOS 300D) was attached to the eyepiece of the microscope. Samples of a non-porous silk film (control) and macroporous silk inverse opal were placed in turn at the focal point, wetted with water and imaged immediately. The non-porous textureless silk film was prepared by deposition of a regenerated fibroin solution ( $8 \% \mathrm{wt}, \mathrm{ca}$. $1 \mathrm{~mL}$ ) onto a mica surface followed by drying in a sealed petri dish for 4 days under ambient conditions. Ethanol $(10 \mathrm{~mL})$ was applied to the film to induce $\beta$-sheet formation, and decanted after 1 hour. Toluene $(10 \mathrm{~mL})$ was also applied for the same duration and then decanted. The silk film was then dried in air and then removed from the mica substrate. 
1 E. Dujardin and S. Mann, Adv. Mater., 2002, 14, 775.

2 D. P. Knight and F. Vollrath, Philos. Trans. R. Soc. London, Ser. B, 2002, 357, 155

3 E. L. Mayes, F. Vollrath and S. Mann, Adv. Mater., 1998, 10, 801

4 Q. Xu, J. Li, Q. Peng, L. Wu and S. Li, Mater. Sci. Eng., B, 2006, 127, 212.

5 J. He and T. Kunitake, Chem. Mater., 2004, 16, 2656.

6 M. Wang, H.-J. Jin, D. L. Kaplan and G. C. Rutledge, Macromolecules, 2004, 37, 6856.

7 J.-H. Yeo, K.-G. Lee, Y.-W. Lee and S. Y. Kim, Eur. Polym. J., 2003, 39, 1195.

8 K. Yamaura, T. Tanigami and S. Matsuzawa, J. Colloid Interface Sci., $1985, \mathbf{1 0 6}, 565$

9 V. Karageorgiou, M. Tomkins, R. Fajardo, L. Meinel, B. Snyder, K. Wade, J. Chen, G. Vunjak-Novakovic and D. L. Kaplan, J. Biomed. Mater. Res., A, 2006, 78, 324.

10 H. J. Kim, H. S. Kim, A. Matsumoto, I. J. Chin, H. J. Jin and D. L. Kaplan, Aust. J. Chem., 2005, 58, 716.
11 U. J. Kim, J. Park, H. J. Kim, M. Wada and D. L. Kaplan, Biomaterials, 2005, 26, 2775.

12 B. Zuo, L. Dai and Z. Wu, J. Mater. Sci., 2006, 41, 3357.

13 C. Holland, A. E. Terry, D. Porter and F. Vollrath, Polymer, 2007, 48, 3388.

14 O. D. Velev and E. W. Kaler, Adv. Mater., 2000, 12, 531.

15 C. W. P. Foo, E. Bini, J. Hensman, D. P. Knight, R. V. Lewis and D. L. Kaplan, Appl. Phys. A: Solid Surf., 2006, 82, 223.

16 D. Wilson, R. Valluzzi and D. Kaplan, Biophys. J., 2000, 78, 2690.

17 X. Chen, Z. Shao, D. P. Knight and F. Vollrath, Proteins, 2007, 68, 223.

18 X. Peng, Z. Shao, X. Chen, D. P. Knight, P. Wu and F. Vollrath, Biomacromolecules, 2005, 6, 302.

19 K. D. Hermanson, D. Huemmerich, T. Scheibel and A. R. Bausch, Adv. Mater., 2007, 19, 1810.

20 N. J. Shirtcliffe, G. McHale, M. I. Newton, G. Chabrol and C. C. Perry, Adv. Mater., 2004, 16, 1929

21 R. Blossey, Nat. Mater., 2003, 2, 301.

22 W. Barthlott and C. Neinhuis, Planta, 1997, 202, 1.

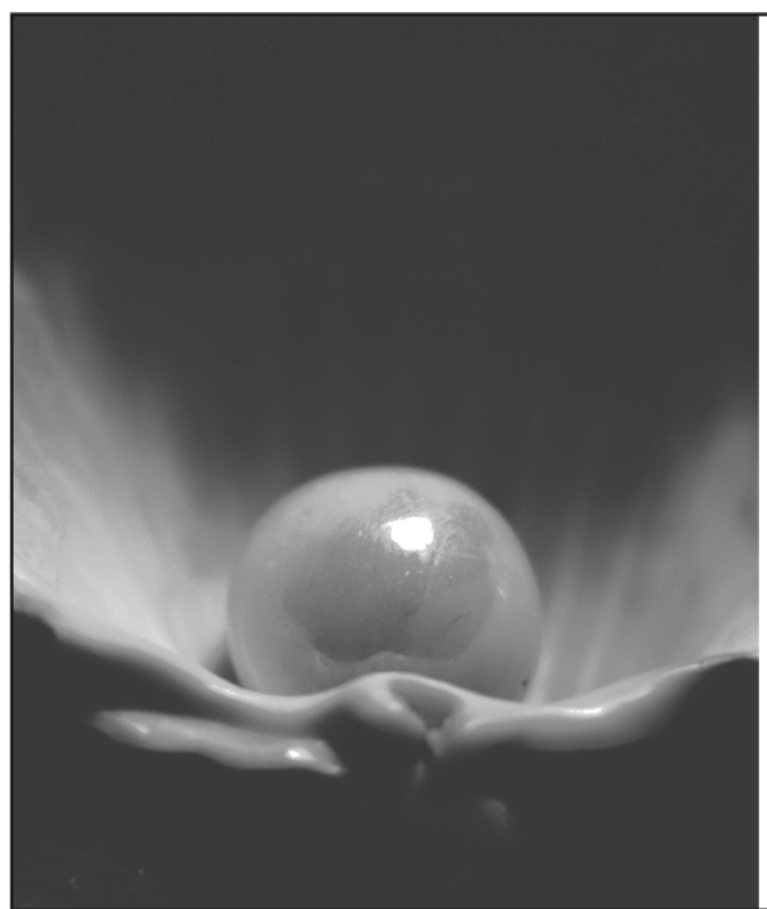

\section{Looking for that Special chemical science research paper?}

\section{TRY this free news service:}

\section{Chemical Science}

- highlights of newsworthy and significant advances in chemical science from across RSC journals

- free online access

- updated daily

- free access to the original research paper from every online article

- also available as a free print supplement in selected RSC journals.*

${ }^{*}$ A separately issued print subscription is also available. 